APS/123-QED

\title{
Guiding of Relativistic Laser Pulses by Preformed Plasma Channels
}

\author{
C.G.R. Geddes, ${ }^{1, *}$ Cs. Toth, ${ }^{1}$ J. van Tilborg, ${ }^{1, \dagger}$ E. \\ Esarey, ${ }^{1}$ C.B. Schroeder, ${ }^{1}$ J. Cary,${ }^{2, \ddagger}$ and W.P. Leemans ${ }^{1, \S}$ \\ ${ }^{1}$ Lawrence Berkeley National Laboratory, \\ 1 Cyclotron Rd, Berkeley CA 94720 \\ ${ }^{2}$ Tech-X Corporation, 5621 Arapahoe Ave Ste. A, Boulder CO 80303
}

(Dated: December 10, 2004)

\begin{abstract}
Guiding of relativistically intense $\left(>10^{18} \mathrm{~W} / \mathrm{cm}^{2}\right)$ laser pulses over more than 10 diffraction lengths has been demonstrated using plasma channels formed by hydrodynamic shock. Pulses up to twice the self guiding threshold power were guided without aberration by tuning the guide profile. Transmitted spectra and mode images showed the pulse remained in the channel over the entire length. Experiments varying guided mode power and simulations show a large plasma wave was driven. Operating just below the trapping threshold produces a dark current free structure suitable for controlled injection.

PACS numbers: Valid PACS appear here
\end{abstract}

\footnotetext{
${ }^{*}$ Also at University of California, Berkeley

${ }^{\dagger}$ Also at Technische Universiteit Eindhoven, the Netherlands

$¥$ Also at University of Colorado, Boulder

$\S$ Electronic address: wpleemans@lbl.gov; URL: http://loasis.lbl.gov
} 
Controlling the propagation of intense laser pulses is important to many applications including harmonic generation [1], laser fusion [2], and laser plasma wakefield accelerators [3]. In laser plasma accelerators, the pondermotive force of an intense laser pulse drives a plasma wave (wake), and particles have been accelerated with gradients of hundreds of $\mathrm{GV} / \mathrm{m}[4-6]$. Peak energies have been limited by the short Rayleigh range, $Z_{R} \approx \pi w_{0}^{2} / \lambda$ where $w_{0}$ is the laser spot size and $\lambda$ the wavelength, over which the laser remains intense [7]. It has been demonstrated theoretically that performance of such accelerators can be greatly enhanced by guiding the drive laser pulse [8-10], which must be at or above an intensity $10^{18} \mathrm{~W} / \mathrm{cm}^{2}$ to produce high accelerating fields. To reach $\mathrm{GeV}$ energies, cm-scale acceleration distances are needed. This is beyond $Z_{R}$ for reasonable laser powers and optimal spot sizes, requiring guiding of the laser pulse to optimize perfomance. Recent experiments have also shown that acceleration of the electron bunch over long distances resulted in the production of high quality electron bunches with narrowed energy distributions and increased energies, even when controlled injection was not used [11-13], and this has also been observed in simulations $[11,14,15]$. By maintaining the laser intensity over many $Z_{R}$, controlled guiding can also allow efficient production of a long, high gradient wake structure without self trapping, allowing the use of controlled injection schemes which may produce very high quality beams [16-18]. Hence, guiding of relativistically intense pulses is essential to obtaining high energy, low emittance, low energy spread electron beams from high gradient laser wakefield accelerators.

Guiding at high intensity requires controlling both diffraction and relativistic modification of the plasma refractive index [19]. Relativistic self guiding occurs when the laser is of high enough power that the quiver motion of the electrons causes their mass to increase, changing the refractive index [20-23]. This effect, which occurs in regimes of interest to wakefield acceleration, provides some self guiding but is unstable, eventually destroying the pulse and limiting propagation distance $[7,19,23]$. Relativistic self guiding is least effective for pulses not much longer than the plasma period, [24], as used for most laser acceleration experiments, limiting acceleration distance to $\approx Z_{R}$ [7]. Previous controlled guiding experiments have demonstrated guiding for input pulse intensities at up to $2 \times 10^{17} \mathrm{~W} / \mathrm{cm}^{2}$, where relativistic effects are unimportant and a parabolic transverse density profile can be matched to guide the low intensity pulse [25-29]. Guiding of high intensity pulses where compensation for self guiding is important has, however, not been studied experimentally. 
In this Letter, we report that laser pulses have been guided for the first time at relativistic intensities over many $Z_{R}$ using plasma channels formed by hydrodynamic shock. The channel has been tuned to guide intensities up to $4 \mathrm{TW}$ (2 times the self guiding threshold) without distortion of the laser mode, and to compensate for relativistic self guiding. This allowed relativistic intensities above $10^{18} \mathrm{~W} / \mathrm{cm}^{2}$, or $a_{0}>1$ (where the normalized vector potential $\left.a_{0}=e E / m \omega c=\gamma v / c\right)$, at the guide output. These pulses are in the relevant regime for laser wakefield acceleration $[8,9]$. The spectrum of the transmitted pulse and analysis of the plasma shape show that the pulse remains in the channel over its entire length. Experiments and simulations show that a large plasma wave is driven by the laser pulse. By allowing drive laser pulses to propagate over many $Z_{R}$ in the plasma, the acceleration distance and hence the electron beam energy and quality of plasma based laser accelerators can be dramatically improved [11, 14, 15], and controlled injection experiments [16-18] may be possible.

The plasma channel was created by two laser pulses in a variant of the ignitor-heater method [26], using the multi arm l'OASIS Ti:Sapphire laser [30, 31] operating at $800 \mathrm{~nm}$ with chirped pulse amplification [32]. A supersonic hydrogen gas jet provided a nearly uniform $2.4 \mathrm{~mm}$ long gas target at an atomic density of $3 \times 10^{19} \mathrm{~cm}^{-3}$. A $60 \mathrm{fs}, 15 \mathrm{~mJ}$ ignitor pulse was focused at $\mathrm{f} / 15$ near the downstream edge of the gas jet. The focal location and $\mathrm{f} /$ number were chosen so that beam convergence roughly balanced ionization induced refraction [33, 34], producing a cylindrical plasma of nearly uniform radius. This plasma was subsequently heated to tens of $\mathrm{eV}$ by inverse Bremsstrahlung using $\sim 50 \mathrm{~mJ}$ from a $250 \mathrm{ps}, 150 \mathrm{~mJ}$ pulse cylindrically focused and incident on the jet from the side. Use of two channel formation pulses allowed both efficient ionization (requiring a high intensity pulse) and heating (optimized with a longer, low intensity pulse) [26]. The resulting hot plasma filament on axis expanded outward, driving a shock wave in the surrounding neutral gas. This shock resulted in a density depletion on axis and a nearly parabolic transverse density profile [35]. By choosing the timing of the guide pulses, and by setting plasma temperature (which determines shock velocity) by adjusting the heater energy, the density profile has been matched to guide the drive pulse. The drive pulse was collinear with the ignitor and focused near the entrance of the channel, with a 55 fs pulsewidth FWHM (full width half max, intensity), powers of 0-9 TW and a focused spot size of $7 \mu \mathrm{m}$ FWHM. This arrangement is shown in Fig. 1.

Interferometry using a frequency doubled (405 nm wavelength) pulse, incident from the 
side, was used to characterize the plasma and to measure its temporal evolution with fs resolution. To optimize viewing of the long narrow plasma channels, an imaging system using cylindrical optics provided differing magnification in the two axes, with a resolution of $17 \mu \mathrm{m}$ (axial) $\times 4 \mu \mathrm{m}$ (transverse). The phase map from the interferometer was Abel inverted to recover the electron density (Fig. 2a,b) assuming a cylindrically symmetric distribution. Symmetry is confirmed by the mode structure of the guided laser pulse (below). The axial density was within $10 \%$ of $1.4 \times 10^{19} \mathrm{~cm}^{-3}$ over the central $1.7 \mathrm{~mm}$ of the jet. The length of the wake drive laser pulse was thus longer than the linear plasma period, providing operation in the self-modulated regime [22]. This allows the channels to extend laser propagation distance and enhance the acceleration performance in current wakefield accelerators, which operate in the self modulated regime to allow self trapping [10, 11]. The profile of the channel shown in Fig. 2 had a 40\% reduced rise in density over a laser spot size compared to the matched profile for a low intensity pulse [26]. This detuning was required to compensate for the presence of self guiding at $4 \mathrm{TW}$, or twice the self guiding threshold $P_{\text {crit }}$ at this density (see below).

In order to characterize the effectiveness of the guide in maintaining the laser mode, a mirror inserted into the beamline and an f/10 achromatic lens imaged the mode directly onto a CCD (mode imager) camera. The lens could be translated to image the entry or exit of the channel. Because of mounting constraints on its $\mathrm{f} /$ number, the mode imager resolution was slightly larger than the focused spot size of the laser so that only a lower limit on guided intensity is obtained from the image intensity. To overcome this problem, the resolution and imaging properties of this system were characterized using known laser spots which were independently measured with a high resolution camera, and also with backlit standard resolution targets. The imaging properties were consistent with diffraction for a circular aperature at f/10 [36], which allowed deconvolution of spot sizes for laser modes that are near the resolving power of the lens. An optical beam splitter was used to send part of the laser energy to an optical spectrometer, allowing simultaneous analysis of the transmitted laser spectrum. Trapped and accelerated electrons, if present, were detected using an integrating current transformer (detection threshold $10^{9}$ electrons).

Propagation of the drive pulse in the plasma was analyzed using an interferometer as well as the mode imager and the spectrometer. The interferometer image of the plasma when the drive pulse propagated in the channel at 4 TW (Fig. 2c) is very similar to that of the 
channel alone (Fig. 2a). The absence of additional plasma ionized outside the channel when the drive pulse is on demonstrates good confinement and minimal leakage over the length of the guide, as laser energy leaked outside the guide would ionize additional plasma. By contrast, the unguided drive beam diffracts rapidly, creating a larger plasma which expands as the laser propagates (Fig. 2d).

Using the channel tuned as described above, the guided laser mode for an input power of $4 \mathrm{TW}$ and intensity of $7 \times 10^{18} \mathrm{~W} / \mathrm{cm}^{2}$ showed no detectable aberration or change in spot size when compared to the input mode (Fig. 3b,a). This demonstrates control of laser propagation over more than 10 diffraction ranges at a power 2 times $P_{\text {crit }}$, and at an intensity useful for acceleration. The output mode was cylindrically symmetric, indicating that the assumption of channel symmetry (above) was valid. The effectiveness of the guide can be seen by comparison to the vacuum propagated mode which displayed diffraction, and to the beam propagated through the gas jet without channeling where diffraction was further increased by ionization effects (Fig. 3c,d). The energy transmission was near 35\%. Deconvolution of the image using the measured imaging system response to various spot sizes (above) indicated that since input and output measured mode sizes were identical, the output mode was within $1 \mu \mathrm{m}$ of the $7 \mu \mathrm{m}$ input, yielding an output intensity of $2.5 \times 10^{18}$ $\mathrm{W} / \mathrm{cm}^{2}$ (the lower limit on intensity from the direct image gives $I>1 \times 10^{18} \mathrm{~W} / \mathrm{cm}^{2}$ ). For a pulse long compared to $\lambda_{p}$, linear fluid theory predicts self guiding at this power without a channel, though this is subject to instabilities which can disrupt propagation [19]. For the $\leq 10 \lambda_{p}$ long pulses used in these experiments however, self guiding alone was never sufficient to guide the pulse over $10 Z_{R}$, and in fact the detuning of the guide required to guide the high intensity pulses was modest, indicating the importance of channeling even for high power beams.

Adjusting channel plasma profiles allowed guiding of modes at various powers by compensating for the changing effect of relativistic self guiding. Fig. 2 and 3 showed guiding of a 4 TW pulse for which the channel profile was flattened, relative to the low power tune, to allow for the relativistic contribution. Fig. 4 shows the guiding of modes at various input powers by a channel which has been tuned to guide a low power $\left(0.5 \mathrm{TW}<P_{\text {crit }}\right)$ beam. Energy throughput is up to $50 \%$ at $0.5 \mathrm{TW}$, compared to $35 \%$ at $4 \mathrm{TW}$, indicating that a substantial amount of the laser energy is depleted into the wake at high powers. The low power beam is well guided by this channel, while pulses exceeding $P_{\text {crit }}$ are distorted. 
Indeed, the guided intensity flat tops for input power $>P_{\text {crit }}$. This indicates the importance of tuning the channel profile to compensate for the focusing effect of relativistic self guiding, and demonstrates that this can be done for $P \geq 2 P_{\text {crit }}$. Above this power, pulses have been guided up to $9 \mathrm{TW}$, and the interferometer shows good confinement of the laser energy at all powers. However, at this power level some distortion of the mode was always observed, with typical output spots near $24 \mu \mathrm{m}$, probably due to the very strong self focusing and coupling to plasma waves in this case $[11,19]$.

To separate the effects of channeling and ionization, mode transmission of the high power beam in a pre-ionized plasma without a channel was also studied. The ignitor beam was fired 80 ps before the main beam, pre-ionizing a plasma $80 \mu \mathrm{m}$ in diameter. The plasma does not expand significantly over 80 ps, so that the plasma had a flat density profile and hence no guiding properties. Propagation was similar in pre-ionized and unionized gas jets indicating that channeling, and not ionization, was responsible for the observed difference in propagation. This is reasonable since for the laser pulse intensities used, the gas is ionized by the foot of the laser pulse at $I<10^{-4} I_{0}$ and hence should not affect propagation of the bulk of the pulse.

The transmitted spectrum of the laser light serves as a check of the channeling efficiency and a diagnostic of plasma wave production. An intense laser pulse propagating in an unionized gas suffers a blue shifting in spectrum due to the time dependent index of refraction as the gas is ionized by the laser [37]. Hence the extent of such ionization blue shifting is an indicator of the laser escaping confinement in the preformed channel. On the other hand, coupling of the laser to plasma waves (as used for laser plasma accelerators) results in red shifting of the laser pulse as energy is depleted into the plasma wave. Fig. 5 shows the normalized transmitted spectrum at 4 TW for the vacuum, unchanneled, and channeled case. In the unchanneled case, nearly all of the laser energy is blue shifted, and transmission of the $800 \mathrm{~nm}$ fundamental is a few percent. In contrast, the channeled spectrum shows a very small blue shifted shoulder, likely caused by residual neutral gas at the channel ends. The transmission of the $800 \mathrm{~nm}$ feature is $\approx 30 \%$, compatible with the transmission numbers from the mode imager, indicating that the transmitted beam is largely unaberrated spectrally as well as spatially. Since the input pulse is nearly transform limited, this also indicates that the pulse remained reasonably coherent temporally. A red shifted shoulder can also be seen beginning to appear in the channeled case, and the energy in this shoulder 
increased to more than $20 \%$ of the fundamental with increasing laser intensity, indicating coupling to plasma waves.

The presence of an intense plasma wave was verified by observing the trapping and acceleration of electrons. For drive pulse powers above 4TW, electrons were self trapped and accelerated [11], indicating that the wave is close to the wave breaking field at 4TW. Electron energies of up to $150 \mathrm{MeV}$ were obtained at $9 \mathrm{TW}$. At intensities just below the trapping threshold, where no electrons are self trapped but laser intensity and hence plasma wave amplitude were maintained over a long scale length, the wave produced would enable experiments on controlled injection into laser wakefields. Such experiments have the potential to both stabilize and increase quality of beams from laser accelerators [16, 17], but require a long plasma wake structure without dark current to accelerate the injected electrons, which has not been available until now.

Simulations using the particle in cell code VORPAL [38] have also indicated good coupling of the laser to plasma waves in the channel. Simulations were performed for the experimental densities and laser parameters, using a moving window copropagating with the laser pulse $90 \mu \mathrm{m}$ long in the longitudinal direction by $80 \mu \mathrm{m}$ transversely, with $1800 x 300$ cells and 10 particles per cell. The guided spot size is $\sim 1 \mu \mathrm{m}$ smaller than the input in these simulations, consistent with the experimental observation. The pulse self modulates, exciting a strong plasma wave with a field averaging $\sim 300 \mathrm{GV} / \mathrm{m}$ over the last $0.5 \mathrm{~mm}$ of the guide at 4 TW. Approximately $30 \%$ of the laser energy is depleted into the wave consistent with the experimentally observed decrease in transmission between 0.5 and 4 TW. This amplitude is also consistent with the estimate obtained by equating the energy in the wake to the energy depleted from the laser using within 50\%. This wave is below the self trapping threshold so that there is no dark current, yet would provide energy gains of $\sim 100 \mathrm{MeV}$ (limited by dephasing [9]) for externally injected electrons. Higher energies would be available at lower densities.

In conclusion, experiments have demonstrated guiding of relativistically intense laser pulses over many $Z_{R}$ in plasmas, and tailoring of the radial plasma profile provided guiding without detectable aberration up to several times the relativistic self guiding threshold. Intensities near $10^{19} \mathrm{~W} / \mathrm{cm}^{2}$ have been guided without self injection of electrons. Experiments as well as simulations show that a substantial plasma wave is excited in the channel. This potentially provides a high gradient structure for controlled injection of electrons, which 
offers the possibility of greatly stabilizing and improving the beam quality of laser driven accelerators.

Work supported by the U.S. Dept. of Energy contracts DE-AC03-76SF00098, DEFG03-95ER40926, DE-FG02-01ER41178, DE-FG02-03ER83857,DE-AC03-76SF00098, SciDAC, and NSF grant 0113907. C.Geddes is also supported the by Hertz foundation. C. Geddes acknowledges his faculty advisor Jonathan Wurtele, at UC Berkeley. We appreciate contributions from Gerry Dugan, Jerome Faure, Gwenael Fubiani, Bob Nagler, Kei Nakamura, Ned Saleh, Brad Shadwick, Leon Archambault, Michael Dickinson, Scott Dimaggio, Don Syversrud, Joe Wallig, and Nathan Ybarrolaza. 
[1] A. Rundquist et al., Science 280, 5268 (1998).

[2] M. Tabak et al., Phys. Plasmas 1, 1626 (1994).

[3] T. Tajima and J. M. Dawson, Phys. Rev. Lett. 43, 267 (1979).

[4] A. Modena et al., Nature 377, 606 (1995).

[5] V. Malka et al., Science 298, 1596 (2002).

[6] W. P. Leemans et al., Phys. Rev. Lett. 89, 4802 (2002).

[7] Z. Najmudin et al., Phys. Plasmas 10, 2071 (2003).

[8] E. Esarey et al., IEEE Trans. Plasma Sci. 24, 252 (1996).

[9] W. P. Leemans et al., IEEE Trans. Plasma Sci. 24, 331 (1996).

[10] R. Hubbard et al., Phys. Rev. E 63, 036502/1 (2001).

[11] C. Geddes et al., Nature 431, 538 (2004).

[12] S. P. D. Mangles et al., Nature 431, 535 (2004).

[13] J. Faure et al., Nature 431, 541 (2004).

[14] A. J. W. Reitsma et al., Phys. Rev. ST Accel. Beams 5, 051301 (2002).

[15] F. Tsung et al., accepted for publication in Phys. Rev. Lett. (2004).

[16] D. Umstadter, J. K. Kim, and E. Dodd, Phys. Rev. Lett. 76, 2073 (1996).

[17] E. Esarey et al., Phys. Rev. Lett. 79, 2682 (1997).

[18] G. Fubiani et al., Physical Review E 70, 16402 (2004).

[19] E. Esarey et al., IEEE J. Quantum Electron. 33, 1879 (1997).

[20] A. Litvak, Sov. Phys JETP 30, 344 (1969).

[21] P. Sprangle, C. M. Tang, and E. Esarey, IEEE Trans. Plasma Sci. PS-15, 145 (1987).

[22] E. Esarey, J. Krall, and P. Sprangle, Phys. Rev. Lett. 72, 2887 (1994).

[23] W. B. Mori, IEEE J. Quantum Electron. 33, 1942 (1997).

[24] P. Sprangle et al., Phys. Rev. Lett. 69, 2200 (1992).

[25] C. G. Durfee III and H. M. Milchberg, Phys. Rev. Lett. 71, 2409 (1993).

[26] P. Volfbeyn, E. Esarey, and W. Leemans, Phys. Plasmas 6, 2269 (1999).

[27] K. Kim et al., in AIP Conference Proceedings (PUBLISHER, ADDRESS, 2002).

[28] E. Gaul et al., Applied Physics Letters 77, 4112 (2000).

[29] Y. Ehrlich et al., Phys. Rev. Lett. 77, 4186 (1996). 
[30] W. P. Leemans et al., Phys. Plasmas 5, 1615 (1998).

[31] C. Toth et al., in SPIE Proceedings 5448 (PUBLISHER, ADDRESS, 2004), in press.

[32] D. Strickland and G. Mourou, Optics Communications 56, 219 (1985).

[33] W. P. Leemans et al., Phys. Rev. A 46, 1091 (1992).

[34] R. Rankin, C. Capjack, and N. B. an P.B. Corkum, Optics Letters 16, 835 (1991).

[35] H. M. Milchberg et al., Phys. Plasmas 3, 2149 (1996).

[36] B. Saleh and M. Teich, Fundamentals of Photonics (Wiley, New York, 1984), p. 128.

[37] E. Esarey, G. Joyce, and P. Sprangle, Phys. Rev. A 44, 3908 (1991).

[38] C. Nieter and J. Cary, J. Comp. Phys. 196, 448 (2004). 
FIG. 1: Experimental arrangement. A plasma was formed by an ignitor pulse in a gas jet, then heated by a heater pulse. Hydrodynamic expansion of the plasma formed a channel which guided the relativistically intense drive pulse is focused at its edge. Propagation of the laser was monitored with a side interferometer and mode imager CCD. Charge of the accelerated electron bunch, if any, was measured using an integrating current transformer (ICT).

FIG. 2: The plasma profile at the time of arrival of the drive pulse was measured with an interferometer. The interferogram of the plasma channel (A) was Abel inverted with symmetry about the $\mathrm{z}$ axis to obtain the transverse density profile (B). When the drive pulse propagated in the channel at 4 TW (C), there was no change in the interferogram, indicating good confinement to the channel. Without the guiding channel (D), the drive pulse diffracted, spreading out as it propagated from left to right.

FIG. 3: Mode images of laser propagation at $4 \mathrm{TW}$, or twice the critical power for self focusing. The guided output mode after $2.4 \mathrm{~mm}$ (>10 diffraction ranges) of propagation (B) is indistinguishable from the input mode (A). Vacuum propagation over the same distance results in a large output mode due to diffraction (C). With gas jet on but without the guide diffraction is enhanced (D), indicating that self guiding is not effective. Note enlarged scale in (C),(D).

FIG. 4: Tuning of the channel to compensate for self guiding. The effect of self guiding on mode structure in the channel is shown by varying the input power in a channel tuned to guide low intensity pulses. For pulses at $0.5 \mathrm{TW}$, below the critical power for self guiding, the output mode is undistorted (A). As power is increased to $2 \mathrm{TW}(\mathrm{B})$, some distortion is evident and this becomes more severe as power is further increased to $4 \mathrm{TW}(\mathrm{C})$ and $8 \mathrm{TW}(\mathrm{D})$. The ignitor-heater produced guide was tuned to guide modes up to $4 \mathrm{TW}$ without distortion by adjusting the density profile to account for the presence of self guiding (see Fig. 3). 
FIG. 5: Normalized transmitted optical spectrum of the drive pulse. The drive pulse propagating in the gas jet has most of its energy blue shifted away from the fundamental by ionization, impairing its usefulness for applications. The channeled beam shows almost undistorted propagation, with the appearance of a red shifted side band indicating depletion of some of the laser energy into plasma waves. The absence of significant blue shifting indicates propagation through ionized plasma, and hence that the pulse remains within the channel. 